\title{
Hymenolepis nana-An Emerging Intestinal Parasite Associated with Anemia in School Children from the Bolivian Chaco
}

\author{
Michele Spinicci, ${ }^{1}$ Fabio Macchioni, ${ }^{2}$ Simona Gabrielli, ${ }^{3}$ David Rojo, ${ }^{4}$ Herlan Gamboa, ${ }^{5}$ Ana Liz Villagrán, ${ }^{6}$ Yolanda Vallejos, ${ }^{7}$ \\ Marianne Strohmeyer, ${ }^{1}$ Mimmo Roselli, ${ }^{1}$ Gabriella Cancrini, ${ }^{3}$ Piero Olliaro, ${ }^{8}$ Antonio Montresor, ${ }^{9}$ and Alessandro Bartoloni ${ }^{1 *}$ \\ ${ }^{1}$ Dipartimento di Medicina Sperimentale e Clinica, Università degli Studi di Firenze, Firenze, Italy; ${ }^{2}$ Dipartimento di Scienze Veterinarie, Università \\ degli Studi di Pisa, Pisa, Italy; ${ }^{3}$ Dipartimento di Sanità Pubblica e Malattie Infettive, Università di Roma Sapienza, Roma, Italy; ${ }^{4}$ Escuela de Salud del \\ Chaco Tekove Katu, Gutierrez, Plurinational State of Bolivia; ${ }^{5}$ Facultad Integral del Chaco, Universidad Autónoma Gabriel René Moreno, Camiri, \\ Plurinational State of Bolivia; ${ }^{6}$ Hospital Básico de Villa Montes, Villa Montes, Plurinational State of Bolivia; ${ }^{7}$ Hospital Municipal de Camiri, Camiri, \\ Plurinational State of Bolivia; ${ }^{8}$ Special Programme for Research and Training in Tropical Diseases (TDR), World Health Organization, Geneva, \\ Switzerland; ${ }^{9}$ Department of Control of Neglected Tropical Diseases (NTD), World Health Organization, Geneva, Switzerland
}

\begin{abstract}
Tropical anemia can have multiple causes, whether socioeconomic, dietary, or infectious. In the Bolivian Chaco, soil-transmitted helminthiases (STH), malaria, and Chagas disease are potential infectious causes of anemia among school-aged children (SAC). Following years of preventive chemotherapy with mebendazole, the prevalence of STH among SAC living in that area is now negligible, whereas protozoan infections are still highly prevalent (81\%); Hymenolepis nana is the most frequent intestinal helminth ( 13\%). We present results of hemoglobin $(\mathrm{Hb})$ assessment and the association between parasitic infections and $\mathrm{Hb}$ levels of that SAC population. Overall, 511 SAC (girls:boys ratio 1: 1, mean age 9.4 years [95\% confidence interval $\{\mathrm{Cl}\}$ : 9.3-9.5]) had $\mathrm{Hb}$ levels measured by using a point of care testing (HemoCue $_{\circledast} \mathrm{Hb} 301$ System; HemoCue, Angelhome, Sweden). The prevalence of anemia was 23\% (117/511), with mean and median $\mathrm{Hb}$ level = $12.2 \mathrm{~g} / \mathrm{dL}$ (95\% Cl: 12.1-12.3; range 9.2-15.4 g/dL). By multivariate analysis, $H$. nana infection was associated with an increased risk of anemia (odds ratio $2.9,95 \% \mathrm{Cl}: 1.5-5.7, P=0.002)$. Two samples $(0.5 \%)$ were positive for Trypanosoma cruzi and none for Plasmodium spp. by polymerase chain reaction of the 439 children tested. Anemia is still a concern among SAC living in the Bolivian Chaco. Our findings call for a greater attention to fecal-oral emerging pathogens, such as $H$. nana, and highlight the importance of water, sanitation, and hygiene improvements for disadvantaged population such as those living in the Bolivian Chaco.
\end{abstract}

\section{INTRODUCTION}

Tropical anemia can have multiple causes-iron deficiency being the most common, especially among women of reproductive age and children, whether caused by inadequate iron dietary intake (the main cause) or contributed to by infectious diseases such as malaria and soil-transmitted helminthiases (STH). ${ }^{1}$ Hookworm infection in particular is known to be associated with chronic gastrointestinal blood loss and micronutrients depletion, and thus anemia, chiefly in pregnancy. ${ }^{2}$ Regular deworming through preventive chemotherapy (PC) of at-risk groups (preschool and schoolchildren and women of reproductive age) is expected to reduce worm burden and STH-induced morbidity in the infected individuals and overall prevalence. ${ }^{3}$ In the Bolivian Chaco, a recent crosssectional survey found that the prevalence of hookworm infection in school-age children has dropped from $50 \%$ in the 1990 s to $0.7 \%$ in 2016, and no cases of Ascaris lumbricoides and Trichuris trichiura-likely the result of 30 years of $\mathrm{PC},{ }^{4}$ and this all the while protozoan infections continue to be prevalent ( $81 \%$ of children had at least one) and the minute tapeworm Hymenolepis nana having become the most frequent helminthic infection ( 13\%).

Hymenolepis nana has cosmopolitan distribution, but prevalence is highest among children living in low-income countries with poor Water, Sanitation, and Hygiene standards. ${ }^{5}$ The adult tapeworm parasitizes the mucosal surface of the small intestine. Eggs of $H$. nana are immediately infective when excreted with the stools, but they cannot survive more than 2 weeks in the environment. Transmission does not

*Address correspondence to Alessandro Bartoloni, SOD Malattie Infettive e Tropicali, Azienda Ospedaliero-Universitaria Careggi, Largo Brambilla 3, Firenze 50134, Italy. E-mail: alessandro.bartoloni@unifi.it require an intermediate host and infection can be passed directly from one infected person to another by hand-to-mouth transmission. Ingested eggs release motile embryos, the oncospheres, which invade the small intestinal mucosa, encysting within villi. In 3-4 days, they develop into the larval cysticercoids, and then evaginate, destroying the villi they invaded; attached to the mucosal surface, cysticercoids develop into the adult tapeworm in about 1 month. Cycles of autoinfection, both internal and external, can also occur, sustaining chronic infection. The impact on the health of infected children is still unclear, but the invasive nature of $H$. nana infection is likely to have been overlooked, especially in children with heavy and/or chronic infection. ${ }^{5}$ During the survey carried out in 2016, we also measured hemoglobin $(\mathrm{Hb})$ concentrations and explored the potential association between parasitic infections and anemia.

\section{MATERIALS AND METHODS}

In September 2016, 519 school-aged children (SAC) from nine rural communities of the Bolivian Chaco, a semiarid and sparsely populated region, located in the southeast of the Plurinational State of Bolivia (lat. $17^{\circ} 58^{\prime}-22^{\circ} 20^{\prime} \mathrm{S}$, long. $64^{\circ} 30^{\prime}-58^{\circ} 50^{\prime} \mathrm{W}$ ) were enrolled in a cross-sectional parasitological survey, whose methodology and results have been previously described in detail. ${ }^{4}$ Briefly, a minimum of 50 third to fifth graders, usually 8-11 years old, were enrolled in each school. Stool samples were analyzed for intestinal parasites by direct wet mount and Kato-Katz technique (one thick smear on a single stool sample from each individual), by doubleblinded readers. At the same time, they were all invited to undergo a single measurement of $\mathrm{Hb}$ using a portable $\mathrm{Hb}$ analyzing system (HemoCue ${ }_{\circledast} \mathrm{Hb} 301$ System; HemoCue, Angelhome, Sweden). Of these nine communities, four were in 
the Cordillera Province (Santa Cruz department) and five in the Gran Chaco Province (Tarija department) with 203/242 and 215/269 participants providing a blood sample for $\mathrm{Hb}$ measurement, respectively.

In eight communities ( $N=439$ SAC), it was also possible to collect dried blood spots (DBS), through finger prick on filter paper, to detect Plasmodium spp. and Trypanosoma cruzi by quantitative polymerase chain reaction (qPCR) (qPCR kits; Genesig, United Kingdom). Demographic data were recorded using a standardized questionnaire. Diagnosis and classification of anemia were established according to the World Health Organization guidelines. ${ }^{6}$

Data were analyzed using STATA 11.0 (StataCorp, College Statio, TX). Frequencies, percentages, and means with $95 \%$ confidence intervals $(\mathrm{Cl})$ were calculated. Multivariate analysis was performed by logistic and linear regression, to establish the association of anemia (categorical) and $\mathrm{Hb}$ levels (continuous), respectively, with intestinal parasitic infections (excluding those with prevalence $<1 \%$ ), allowing for the province where the communities were located. Results were considered significant when the $P$ value was $<0.05$.

The study, conducted with the support of the Bolivian Ministry of Health (Convenio Ministerio de Salud, Estado Plurinacional de Bolivia/Cátedra de Enfermedades Infecciosas, Universidad de Florencia, Italia) and the Guaraní political organization (Asamblea del Pueblo Guaraní), was approved by the local Ethic Committee. A written informed consent was obtained by a relative or a legal guardian of each participating child.

\section{RESULTS}

Overall, 511 children had an $\mathrm{Hb}$ measurement (girls:boys ratio 1:1); the mean age was 9.4 years $(95 \% \mathrm{Cl}$ : 9.3-9.5, range $7-16)$. The prevalence of anemia $(\mathrm{Hb}<11.5 \mathrm{~g} / \mathrm{dL})$ was $23 \%$ (117/511), which was mild (11.5-11.0 g/dL) in 54 cases $(46 \%)$ and moderate $(11.0-8.0 \mathrm{~g} / \mathrm{dL})$ in $63(54 \%)$; there was no case of severe anemia $(<8.0 \mathrm{~g} / \mathrm{dL})$. The mean and median $\mathrm{Hb}$ level was $12.2 \mathrm{~g} / \mathrm{dL}$ (95\% Cl: 12.1-12.3; range 9.2-15.4 g/dL).
School-aged childrens living in communities of the Cordillera Province (department of Santa Cruz) had a higher prevalence rate of anemia than those living in the communities of the Gran Chaco Province (department of Tarija) (30\% versus 17\%, respectively; $P<0.001)$ as well as lower median $\mathrm{Hb}$ values $(11.9$ versus $12.4 \mathrm{~g} / \mathrm{dL} ; P<0.001$, Mann-Whitney test). No between-sex statistical differences were found in the frequency of anemia and $\mathrm{Hb}$ levels.

Of the 511 participating children, $418(82 \%)$ also provided a stool sample. The prevalence of anemia in this subset $(21.3 \%$, 89/418) was not statistically different from that of the overall group $(22.9 \%, 117 / 511)(P=0.56)$.

The prevalence of infections and anemia is presented in Table 1: $82 \%$ of children had at least one parasite and $58 \%$ had two or more; the most frequent protozoan species was Blastocystis and the most frequent helminthic species was H. nana.

By multivariate analysis, of all intestinal parasitic infections, only $H$. nana was associated with an increased risk of anemia (odds ratio $2.9,95 \% \mathrm{Cl}: 1.5-5.7, P=0.002$ ) and inversely correlated with the $\mathrm{Hb}$ level $(P=0.016)$. No correlation was found between $H$. nana egg counts and $\mathrm{Hb}$ levels (ageadjusted linear regression) or anemia (age-adjusted logistic regression).

Among 439 DBS analyzed by PCR, two (0.5\%) were positive for $T$. cruzi and none for Plasmodium spp.

\section{DISCUSSION}

Today, approximately one in four to one in five school-age children living in the Bolivian Chaco is anemic, half of them having moderate anemia ( $\mathrm{Hb}$ levels 11 to $8 \mathrm{~g} / \mathrm{dL}$ ). The situation has not improved in the past quarter of a century: a 1990 study carried out in two rural communities of the same area showed rates of anemia of $22 \%$ and $16 \%$, and mean $\mathrm{Hb} 11.8$ and 12.4 $\mathrm{g} / \mathrm{dL}$, among pre-SAC (2-5 years old) and SAC (6-9 years old), respectively. ${ }^{7}$ At that time, STH infections were highly prevalent (41.0-64.5\%), with hookworm prevalence reaching 50\%, $5.6 \%$ of which of heavy or moderate intensity $(>2,000$ eggs

TABLE 1

Risk factor for anemia: univariate and multivariate analysis

\begin{tabular}{|c|c|c|c|c|c|c|}
\hline & \multirow[b]{2}{*}{ Total $N(\%)$} & \multirow[b]{2}{*}{ Anemia* $N(\%) \dagger$} & \multicolumn{2}{|c|}{ Univariate analysis } & \multicolumn{2}{|c|}{ Multivariate analysis } \\
\hline & & & OR $(95 \% \mathrm{Cl})$ & $P$ value & OR $(95 \% \mathrm{Cl})$ & $P$ value \\
\hline Age in years mean $(95 \% \mathrm{Cl})$ & $9.4(9.3-9.5)$ & - & - & - & $0.8(0.6-0.9)$ & $0.01 \ddagger$ \\
\hline Gender female & $256(50)$ & $64(25)$ & - & - & $1.3(0.8-2.1)$ & 0.31 \\
\hline Parasitism (any one infection) & $342(82)$ & $78(23)$ & $1.7(0.9-3.8)$ & 0.11 & - & - \\
\hline Polyparasitism (any two or more infections) & $241(58)$ & $58(24)$ & $1.5(0.9-2.5)$ & 0.11 & - & - \\
\hline \multicolumn{7}{|l|}{ Province } \\
\hline Cordillera (Dep. Santa Cruz) & $242(47)$ & $72(30)$ & $2.1(1.4-3.2)$ & 0.001 & $1.8(1.1-3.0)$ & 0.02 \\
\hline Gran Chaco (Dep. Tarija) & $269(53)$ & $45(17)$ & 1 & - & 1 & - \\
\hline Hymenolepis nana & 49 (12) & $20(41)$ & $3.0(1.5-5.8)$ & $<0.001$ & $2.9(1.5-5.7)$ & 0.002 \\
\hline Entamoeba histolytica complex & $30(7)$ & $5(17)$ & $0.7(0.2-2.0)$ & 0.52 & $1.0(0.3-2.8)$ & 0.97 \\
\hline Giardia intestinalis & $99(24)$ & $21(21)$ & $1.0(0.5-1.8)$ & 0.98 & $0.9(0.5-1.6)$ & 0.74 \\
\hline Blastocystis spp. & $209(50)$ & $54(26)$ & $1.7(1.0-2.9)$ & 0.02 & $1.6(0.9-2.6)$ & 0.08 \\
\hline Entamoeba nana & $110(26)$ & $28(25)$ & $1.4(0.8-2.4)$ & 0.21 & $1.2(0.7-2.2)$ & 0.48 \\
\hline Entamoeba coli & $111(27)$ & $27(24)$ & $1.3(0.7-2.2)$ & 0.36 & $1.2(0.7-2.2)$ & 0.45 \\
\hline Entamoeba hartmanni & $118(28)$ & $27(23)$ & $1.1(0.7-1.9)$ & 0.62 & $1.0(0.6-1.8)$ & 0.96 \\
\hline lodamoeba bütschlii & $24(6)$ & $3(13)$ & $0.5(0.1-1.8)$ & 0.28 & $0.3(0.1-1.2)$ & 0.09 \\
\hline
\end{tabular}

$\mathrm{Cl}=$ confidence interval; $N=$ number; $\mathrm{OR}=$ odds ratio. Significant associations are highlighted in bold. Overall, 117 cases of anemia (23\%) were detected of 511 school-aged children enrolled in the study; among them, 418 provided a fecal sample. Parasites detected at less than $1 \%$ prevalence were arbitrarily excluded from the model (Hookworm: $0.7 \%$; Enterobius vermicularis: $0.7 \%$; Chilomastix mesnili: 0.5\%; Ascaris lumbricoides, Trichuris trichiura, Strongyloides stercoralis: no cases).

*The cutoff for the diagnosis of anemia was $11.5 \mathrm{~g} / \mathrm{dL}$ in the age group $7-11$ and $12 \mathrm{~g} / \mathrm{dL}$ in $12-14$.

t Row percentage.

$\ddagger$ Risk of an anemia decreases with age (OR 0.8 for each 1 -year increase). 
per gram), and T. trichiura prevalence ranged from $14.5 \%$ to $19.3 \%$ (all of light intensity). ${ }^{8}$ Today, STH prevalence is $<1 \%$, yet this dramatic decrease does not seem to have modified the risk of anemia in school-age children in the past three decades (16\% in 1990 versus $23 \% 2016$, Pearson's $X^{2}$ test $P=0.20$ ), possibly because STH infections were of low intensity in 1990. ${ }^{4}$ Yet, still today more than $80 \%$ of children have at least one intestinal parasitic infection and nearly $60 \%$ have two or more, essentially protozoa; about every other child is infected with Blastocystis spp., and one in four is infected with an Entamoeba species. Protozoan infections do not seem to be associated with anemia.

We found that today the most prevalent helminth species is $H$. nana (a situation shared with other reports from India, Africa, and Latin America) and that $H$. nana infection is associated with anemia and decreased $\mathrm{Hb} .{ }^{9-11}$ Despite it emerging as a leading infection in children in tropical areas, our knowledge of the impact of $H$. nana infection on their health is limited. Symptoms that have been attributed to $H$. nana infection in children are generic, such as diarrhea, abdominal pain, irritability, headaches, fever, fatigue and stunting ${ }^{9,10,12}$; only few studies reported an association with anemia. ${ }^{13,14}$ Infection is hand-to-mouth, and autoinfection is common, whereby continuous injury of the intestinal villi is sustained over a long time if untreated, causing enteritis and impaired intestinal permeability, with micronutrient intestinal leakage and low levels of vitamin B12 and folate in blood. ${ }^{15}$ This could explain the lack of correlation between egg counts in the feces and degree of anemia.

Presently, there are no control programs specifically addressing $H$. nana or other tapeworms, although they can be treated, such as schistosomiasis, with a single dose of praziquantel. ${ }^{12,16}$ The persistently high prevalence of anemia in the region is of concern. Clearly socioeconomic conditions in this area have not improved significantly over the past three decades. As for infections, considering that schistosomiasis is absent in this area, we looked for Plasmodium and T. cruzi. By PCR, all 439 samples tested were negative for malaria, possibly because they were collected during the dry season, whereas two of 439 were positive for $T$. cruzi $(0.5 \%)$. This confirms that active transmission of $T$. cruzi continues within this area, where house infestation rates by the vector Triatoma infestans remain high, despite decades long efforts, and hints to the possibility of chronic iron-deficiency anemia following repeat Triatominae bites. ${ }^{17,18}$ Recently, Babesia microti has also been reported in rural population of the Bolivian Chaco. ${ }^{19}$

Poverty and low socioeconomic conditions, which translate into inadequate dietary intake of micronutrients, remain the leading causes of anemia. The imbalance in the prevalence of anemia between SAC living in the Cordillera Province and those from the Gran Chaco Province is probably related to the different socioeconomic levels of the two provinces (poverty rate $60 \%$ versus $38 \%$, respectively). ${ }^{20}$ However, an excess risk of anemia in children infected with $H$. nana emerged, independently from other factors, at a time when hookworms and other soil-transmitted helminths have virtually disappeared in these communities. Because the prevalence of $H$. nana infection is relatively low (12\%), ultimately it is associated with anemia in only $5 \%$ of children in the community.

However, our findings call for a greater attention to $H$. nana, and more broadly to fecal-oral protozoa and helminths as emerging pathogens, especially in areas where STH infections are successfully controlled and other parasitic infections classically related to anemia have declined. Water, Sanitation, and Hygiene interventions are critical to achieve sustainable improvements in health conditions of these population, including anemia. ${ }^{21}$

Received May 9, 2018. Accepted for publication July 21, 2018.

Published online October 8, 2018.

Acknowledgments: We are grateful to Father Tarcisio Ciabatti, Sister Maria Bettinsoli, and Francesco Cosmi (Convenio Ministerio de Salud-Vicariato de Camiri) for their support in carrying out this study, and to the students of the Escuela de Salud del Chaco, Tekove Katu, Gutierrez, for their valuable assistance during the fieldwork. We would like to acknowledge the great work of all the health-care providers, both at the local and central levels, involved in the mass drug administration and PC programme. Last and not least, we would also like to thank the local communities that participated in the study.

Financial support: This work was supported by grants from the Regione Toscana (Italy), Progetti di Iniziativa Regionale (PIR) 2015 ('Supporto al miglioramento della condizione di salute della popolazione del Chaco Boliviano').

Disclosure: P. O. and A. M. are staff members of the WHO. The opinions expressed in this article are those of the authors and may not reflect the views and policies of the WHO.

Authors' addresses: Michele Spinicci, Marianne Strohmeyer, Mimmo Roselli, and Alessandro Bartoloni, Università degli Studi di Firenze, Florence, Italy, E-mails: michele.spinicci@unifi.it, marianne. strohmeyer@unifi.it, mimrose@tin.it, and alessandro.bartoloni@unifi. it. Fabio Macchioni, Università degli Studi di Pisa, Pisa, Italy, E-mail: fabio.macchioni@unipi.it. Simona Gabrielli and Gabriella Cancrini, Università di Roma Sapienza, Rome, Italy, E-mails: simona.gabrielli@ uniroma1.it and gabriella.cancrini@uniroma1.it. David Rojo, Escuela de Salud del Chaco Tekove Katu, Gutierrez, Plurinational State of Bolivia, E-mail: davidrojomayaregua@gmail.com. Herlan Gamboa, Universidad Autónoma Gabriel René Moreno, Camiri, Plurinational State of Bolivia, E-mail: herlan.gamboa@hotmail.com. Ana Liz Villagrán, Hospital Básico de Villa Montes, Villa Montes, Plurinational State of Bolivia, E-mail: ana_liz1971@hotmail.com. Yolanda Vallejos, Hospital Municipal de Camiri, Camiri, Plurinational State of Bolivia, E-mail: yolanda_vallejos@hotmail.com. Piero Olliaro and Antonio Montresor, World Health Organization, Geneva, Switzerland, E-mails: olliarop@who.int and montresora@who.int.

\section{REFERENCES}

1. WHO, 2005. Worldwide Prevalence of Anaemia. Geneva, Switzerland: World Health Organization.

2. Gyorkos TW, Gilbert NL, 2014. Blood drain: soil-transmitted helminths and anemia in pregnant women. PLoS Negl Trop Dis 8: e2912.

3. WHO, 2017. Guideline: Preventive Chemotherapy to Control SoilTransmitted Helminth Infections in At-Risk Population Groups. Geneva, Switzerland: World Health Organization.

4. Spinicci M et al., 2018. Scaling down of a deworming programme among school-age children after a thirty-year successful intervention in the Bolivian Chaco. Trop Med Int Health 23: 616-621.

5. Thompson RC, 2015. Neglected zoonotic helminths: Hymenolepis nana, Echinococcus canadensis and Ancylostoma ceylanicum. Clin Microbiol Infect 21: 426-432.

6. WHO, 2011. Haemoglobin Concentrations for the Diagnosis of Anaemia and Assessment of Severity. Geneva, Switzerland: World Health Organization.

7. Bartoloni A, Guglielmetti $P$, Salazar E, Nicoletti A, Difonzo AR, Roselli M, Anichini P, Cancrini G, 1995. The relationship between haematological measurements and hookworm intensity in children of two rural Bolivian communities. Giornale Italiano di Malattie Infettive 1: 45-50.

8. Bartoloni A, Guglielmetti P, Cancrini G, Gamboa H, Roselli M, Nicoletti A, Paradisi F, 1993. Comparative efficacy of a single 
$400 \mathrm{mg}$ dose of albendazole or mebendazole in the treatment of nematode infections in children. Trop Geogr Med 45: 114-116.

9. Cabada MM, Morales ML, Lopez M, Reynolds ST, Vilchez EC, Lescano AG, Gotuzzo E, Garcia HH, White CA Jr., 2016. Hymenolepis nana impact among children in the highlands of Cusco, Peru: an emerging neglected parasite infection. $A m \mathrm{~J}$ Trop Med Hyg 95: 1031-1036.

10. Mirdha BR, Samantray JC, 2002. Hymenolepis nana: a common cause of paediatric diarrhoea in urban slum dwellers in India. J Trop Pediatr 48: 331-334.

11. Tefera E, Mohammed J, Mitiku H, 2015. Intestinal helminthic infections among elementary students of Babile town, eastern Ethiopia. Pan Afr Med J 20: 1-10.

12. Soares Magalhães RJ, Fançony $C$, Gamboa D, Langa AJ, SousaFigueiredo JC, Clements AC, Vaz Nery S, 2013. Extending helminth control beyond STH and schistosomiasis: the case of human hymenolepiasis. PLoS Negl Trop Dis 7: 1-4.

13. Oliveira D, Ferreira FS, Atouguia J, Fortes F, Guerra A, CentenoLima S, 2015. Infection by intestinal parasites, stunting and anaemia in school-aged children from southern Angola. PLOS One 10: 1-10.

14. Willcocks B, McAuliffe GN, Baird RW, 2015. Dwarf tapeworm (Hymenolepis nana): characteristics in the northern territory 2002-2013. J Paediatr Child Health 51: 982-987.
15. Mohammad MA, Hegazi MA, 2007. Intestinal permeability in Hymenolepis nana as reflected by non invasive lactulose/ mannitol dual permeability test and its impaction on nutritional parameters of patients. J Egypt Soc Parasitol 37: 877-891.

16. Braae UC, Magnussen P, Harrison W, Ndawi B, Lekule F, Johansen MV, 2016. Effect of national schistosomiasis control programme on Taenia solium taeniosis and porcine cysticercosis in rural communities of Tanzania. Parasite Epidemiol Control 3: 245-251.

17. Samuels AM et al., 2013. Epidemiology of and impact of insecticide spraying on Chagas disease in communities in the Bolivian Chaco. PLoS Negl Trop Dis 7: e2358.

18. Schofield CJ, 1981. Chagas disease, triatomine bugs, and bloodloss. Lancet 317: 1316.

19. Gabrielli $S$, Totino $V$, Macchioni $F$, Zuñiga $F$, Rojas $P$, Lara $Y$, Roselli M, Bartoloni A, Cancrini G, 2016. Human babesiosis, Bolivia, 2013. Emerg Infect Dis 22: 1445-1447.

20. Instituto Nacional de Estadística, 2018. Anuario Estadístico. La Paz, Plurinational State of Bolivia: Ministerio de Planificación del Desarrollo.

21. Vilchez Barreto PM et al., 2017. Prevalence, age profile, and associated risk factors for Hymenolepis nana infection in a large population-based study in northern Peru. Am J Trop Med Hyg 97: 583-586. 